William F. Krupke

August 7, 1985

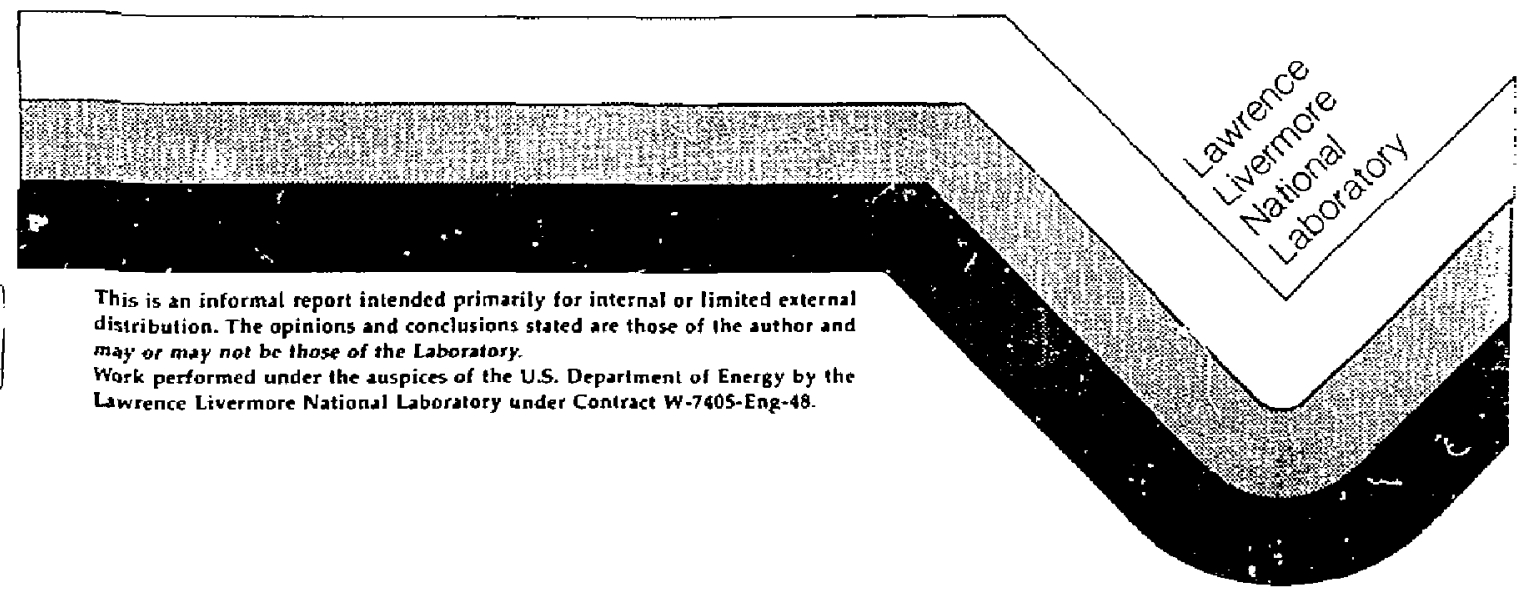

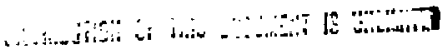




\section{Specific Heat Loading in Nd:Glass Lasers ${ }^{\dagger}$}

by

William F. Krupke

\section{Summary}

The specific thermal load parameter, $x$, for xenon flashlamp-pumped Nd:glass gain media is written as a function of neodymium concertration, pump pulse duration, and energy extraction cfficiency. The currently available data on radiative and nonradiative decay probabilities of several commercial Nd:glasses are used to calculate and graph specific thermal load parameter values. By factoring these results into performance scaling relationships for zig-zag and disk lasers, specific Nd:glasses can be selected for optimized laser performance.

\section{Introduction}

The average pawer capability of repetitively pulsed Nd:glass lasers is directiy related to the specific thermal load parameter. $x$. This parameter is defined as the heat energy density released in the medium during pumping and after extraction, divided by the stored energy density at the time of energy extraction. The magnitude of $x$ depends on the radiative and nonradiative transition probabilities for the relaxation of pump-excited electrons in the glass, on the duration of pump pulse relative to the energy storage lifetime of the medium, and on the degree of gain saturation (or extraction efficiency). Now, in order to 7 imit the temperature rise in the working medium of a high average power solid state laser, relatively thin slabs of glass must be used. "This, in

\footnotetext{
'Aitinur's Note: This UCID is derived from a Lawrence Livermole Naiconal Laboratory Internal Memorandum dated March 30, 1983, WFK 83-129.
} 
turn, dictates the use of high neodymium concentrations in order to achieve a high absorbed fraction of the pump radiation from xenon flashlamps. However, since the nonradiative (heating) rate increases with neodymium ion concentration, one is constrained in neodymium concentration. To optimize laser performance in the face of this constraint, one needs to know the value of the specific thermal load parameter as a function of neodymium ion concentration, normalized pump pulse duration, and extraction efficiency.

\section{Heating Mechanisms}

Heating of Nd:glass takes place when an excited neodymi um ion relaxes from one J-7evel to the next lower lying l-level, with the emission of one or more phonons. (It is properly assumed here that pump radiation is filtered in such a manner that direct heating via UV and IR transitiors of the glass host is precluded. I The mu?tiphonon heating pathways for Nd:glass are shown schematicaliy in Figures 1 and 2. Note that the amount of heating associated with each decay channel is expressed here as a fraction of the energy of a 1060 nin photon $\left(9400 \mathrm{~cm}^{-1}\right)$. Figure 1 shows the multiphonon heating channels operative in the limit of zero neodymium concentration. A xenon flashlamp pump photon, with an average wavelength of $650 \mathrm{~nm}\left(16,000 \mathrm{~cm}^{-1}\right)$, excites a neodymium ion with this average energy. The ion relaxes in about $30 \mathrm{~ns}$ to the upper laser $\mathrm{J}-\mathrm{Tevel}\left({ }^{4} \mathrm{~F}_{3 / 2}\right)$ with the release of 0.426 unics of heat. Based on a limited amount of experimental data, ${ }^{2}$ it is assumed here that the quantum yield of producing an ${ }^{4} F_{3 / 2}$ excited state is urity for excitation in any of the higher lying $\mathrm{Nd}^{3+}$ ion bands. A number of decay pathways for the ${ }^{4} F_{3 / 2}$ ion are operative, each with a different amount of heating associated with $i t$. The con may decay radiatively with probability $A_{4 j}$ to one of the ${ }^{4}$ I J-Tevels by emission of a photon at 880 , 1060, or $1350 \mathrm{~nm}$. These decay processes are immediately followed by nonradiative relaxation to the ${ }^{4} I_{9 / 2} \mathrm{~J}$-level, producing $0.00,0.22$, 0.44 units of heat, respectively. Alternatively, the ${ }^{4} F_{3 / 2}$ ion may relax nonradiatively to the ${ }^{4} I_{15 / 2} \mathrm{~J}-1$ evel by emission of phonons, 
followed by successive photion relaxation through each of the lower ${ }^{4}$ I $\mathrm{d}-\mathrm{l}$ evels to the ground $\mathrm{J}-\mathrm{ievel}$. This totally nonradiative process produces 1.22 units of heat. The average amount of heat produced per ion decaying from the ${ }^{4} F_{3 / 2}$ J-level is the sum of the heat energies per channel, each weighted by the probability of deczy via that channel. The branching ratios for radiative decay are virtually jndependent of glass composition and are $0.40,0.50$, and 0.10 for the 880,1060 , and $1350 \mathrm{~nm}$ transitions, respectively. (The ironching ratio to the ${ }^{4}{ }_{15 / 2}$ $\mathrm{J}$-level is less than 0.005 and can be neglected.) The radiative lifetime of the ${ }^{4} F_{3 / 2} \mathrm{~J}-1$ evel, of course, depends on the glass composition. The transition probability for multiphonon relaxation of the ${ }^{4} F_{3 / 2}$ $\mathrm{J}$-level depends on glass composition and is typicaliy about 200 per second for silicate glasses ${ }^{3}$ and about 500 per second for phosphate glasses. 4,5 In the presence of intense radiation at $1060 \mathrm{~mm}$, the probability that the excited ${ }^{4} F_{3 / 2}$ ion decays by emission of a $1060 \mathrm{~nm}$ photon becomes very large compared to all other decay probabilities, and the ensuing heat fraction is 0.22 units.

When the concentration of neodymium is increased above some relatively small value (typically 0.5 wt-s), an additional heating decay channel becomes increasingly important. Figure 2 shows this heating channel schematically. A neodymium ion in the metastable ${ }^{4} F_{3 / 2}$ J-level interacts with a ground state neadymium ian, producing two neodymium ions in excited ${ }^{4}{ }_{15 / 2} \mathrm{~J}$-levels. The latter ions relax nonradiatively to their ${ }^{4} \mathrm{I}_{9 / 2}$ ground levels by phonon emission. Relaxation of a ${ }^{4} F_{3 / 2}$ ion by this process results in the production of 1.22 units of heat. The rate of heating via this quenching mechanism, $W_{q}(\rho)$, depends on the concentration of neodymium jons as we 11 as on the composition of the glass.

Dependence on Nomalized Pump Pulse Duration

For purposes of illustration, consider the case of the Nd:glass medium being pumped with a constant pump intensity, $P$, for a duration, 
$\tau_{p}$. Let the medium be characterized by a fluorescence lifetime,

$\tau_{f}$. The ${ }^{4} F_{3 / 2}$ ion density at any time is given by

$$
\begin{array}{ll}
n_{4}(t)=P \tau_{f}\left[1-e^{-t / \tau_{f}}\right] ; & 0 \leq t \leq \tau_{p} \\
n_{4}(t)=P \tau_{f}\left[1-e^{-\tau_{p} / \tau_{f}}\right] e^{\tau_{p} / \tau_{f}} e^{-t / \tau_{f}} ; & \tau_{p} \leq t
\end{array}
$$

Since the total number of ${ }^{4} F_{3 / 2}$ ions produced during time, $t$, is simply $P t$, the fraction of ${ }^{4} F_{3 / 2}$ ions remaining at time, $t$, is

$$
\begin{array}{ll}
f_{r}=\left(\tau_{f} / t\right)\left[1-e^{-t / \tau_{f}}\right] ; & 0 \leq t \leq \tau_{p} \\
f_{r}=\left(\tau_{f} / t\right)\left[1-e^{-\tau_{p} / \tau_{f}}\right] e^{\tau_{p} / \tau_{f}} e^{-t / \tau_{f}} ; \tau_{p} \leq t
\end{array}
$$

In practice, energy is extracted at time $t=\tau_{p}$ before fluorescence decay decreases the achieved excited population. The fraction remaining at $t=\tau_{p}$ is

$$
f_{r}\left(\tau_{p}\right)=\left(\tau_{f} / \tau_{p}\right)\left[1-e^{-\tau_{p} / \tau_{f}}\right]
$$

which is graphed as a function of the normalized pump pulse duration $\tau_{p} / \tau_{f}$ in Figure 3 . For comparison, the more realistic case of a half-period sine pump puise, calculated by Trenholme, is al so shown. Over the range of practical interest, the half-period sine pulse shape predicts a fraction stored which is $0-20$ percent 1 arger than that predicted by the square pump pulse shape. 


\section{Specific Thermal Load Parameter}

If we let $\eta_{\text {ext }}$ equal the extraction efficiency (defined as the number of $1060 \mathrm{~nm}$ photons extracted per unit valume divided by the number of $7060 \mathrm{~nm}$ photon equivalence stored per unit volume), we can write the specific thermal load parameter as the sum of four terms:

$$
x=\frac{1}{f_{r}}\left\{0.426+H(0)\left[1-f_{r}\right]+n_{\text {ext }} f_{r}(0.22)+H(0)\left(1-n_{e x t}\right) f_{r}\right\}
$$

The first term gives the heat fraction generated in producing ${ }^{4} F_{3 / 2}$ ians. The second term gives the incremental heating fraction which occurs due to spontaneous decay during pumping. The third tem gives the he: fraction generated by the fraction of ions winichi undergo stimulated emission at the end of the pump pulse. The fourth term gives the fractional heat generated by the unextracted ions as they decay spontaneously back to equilibrium. In this expression, the quantity $H(\rho)$ is defined by

$$
H(\rho)=\frac{\sum_{j=0}^{2} A_{4 j} \Delta E_{j 0}+\Delta E_{40}\left[W_{43}+W_{q}(\rho)\right]}{\sum_{j=0}^{2} A_{4 j}+W_{43}+W_{q}(\rho)}
$$

where $p$ is the neodymium concentration and the other parameters are defined in Figures 1 and 2 . This equation expresses the decay-probability-weighted sum of the heat fractions for the various decay pathways shown in Figures 1 and 2 .

The concentration dependent decay probabilities and values for $H(0)$ are summarized in Tables 1-5 for two silicate (ED-2, LG-650) and three phosphate (LHG-5, LG-750, S-16) glasses. It should be noted that both radiative and nonradiative transition probabilities vary from site th 
site in a given glass ${ }^{3}$ and that one must adopt some appropriate average values for the ensemble of excited ions. The silicate glasses tend to have a fairly wide variation of radiative and nonradiative rates and a fairly low ${ }^{4} \mathrm{~F}_{3 / 2}$ multiphonon decay rate, $W_{43}$, of about 200 per second. 3,5 The phosphates tend to have a narrower variation of transition probability values, but are reported to possess a fajrily high ${ }^{4} F_{3 / 2}$ multiphonon decay rate, $W_{43}$, of 500 per second, 4,5 Many of the phosphate glasses studied at LLNL are reported ${ }^{6-8}$ to have measured ${ }^{4} \mathrm{~F}_{3 / 2}$ fluorescence 1 ifetimes significantly in excess of the calculated radiative lifetimes. This is not only internaliy inconsistent, but it is also at odds with the presence of a significant multiphonon decay probability. In Tables 3-5, 1 have adopted $W_{43}$ multiphonon decay rates of 260 per secand for LHG-5, 145 per second for LG-750, and zero per second for S-16. Radiative rates are based on the best, low-water-content data available. 7,8 However, the specific thermal load parameters may have to be significantly revised if more accurate measurements show that ${ }^{4} F_{3 / 2}$ multiphonon decay rates are substantially larger than those adopted here, as is suggested by the Soviet work. ${ }^{4}$ With the aforementioned caveats, the data from these tables are used to construct the specific thermat Toad paraneter plots shown in Figures 4-7.

Figure 4 shows $x$ as a function of neodymium ion concentration for a nomalized pump pulse duration of 2 (upper cluster of curves) and of 0.7 (lower cluster of curves). Parameter values range from a low of about 0.8 to a high of about 2. For all the glasses save LG-650, $x$ varies a7most linearly with neodymium concentration. LG-650 exhibits a relatively complex dependence of $x$ on necdymium concentration, dictated by its low radiative transition probabilities and its curious concentration quenching dependence (the latter may be an artifact of inaccurate fluorescence lifetime data). 
Figure 5 shows $x$ as a function of nonmalized pump pulse duration for an assumed extraction efficiency of 0.5 and a fixed neodymium concentration of 6 wt-\% in all glasses. The numbers shown aiong the curves give the pump pulse durations in microseconds. The panel at the top of Figure 5 reminds us of the fraction stored (for a lalf-period sine pulse shape) as a function of the normalized pump pulse duration. All of the glasses are well behaved, with a little "kink" occurring near unity pump pulse duration; nc fundamental physical reason is readily obvious for this behavior, but the effect is of little consequence.

Figure 6 illustrates the dependence of $x$ on extraction efficiency, $n_{\text {ext }}$, assuming a nomalized purap pulse duration of 0.7 (fraction storad of 0.78 ) and a neodymium concentration of 6 wt- $\%$ in all glasses. As noted previcusiy, a high degree of gain saturation preferentially drives the excited electrons into the terminal ${ }^{4}{ }_{11 / 2} \mathrm{~J}-1 e v e 1$. Upun relaxation, this level produces relatively little heating $(0.22$ units/icn). High efficiency extraction thus "short circuits" the ion-ion quenching decay channel (with 1.22 units/ion), following energy extraction. Since ion-ion quenching and heating increases with neodymi um concentration, we would expect high extraction efficiencies to be most effective in iimiting the heating rate at high neodymium concentrations. This effect is shown in Figure 7, which plots the derivative of $x$ with respect to extraction efficiency, as a function of neodymium concentration. (A normalized pump plise duration of 0.7 , corresponding to a fraction stored of 0.78 , is assumed.) Near a concentration of one wt- $\%$, the $x$ parameters for phosphates exhibit virtualiy no dependence on extraction efficiency. However, at the higher concentrations, significant suppression of heating occurs at higher extraction efficiencies. The curves shown can be used to estimate the magnitude of a thermal gradient which would be imprinted in a glass slab or disk due to spatial variation of the intensity of an extraction beam. 
Using Equations (6) and (7) and the data in Tables 1-5, other cross-cuts of the dependence of $x$ on neodymium concentration, nomalized pump pulse duration, and extraction efficiency can be generated.

\section{Comparisan with Experiment}

I know of only one (indirect) measurement of the specific thema? load parameter in a repetitively pulsed Nd:glass laser. J. Eggleston and B. Byer of Stanford have reported ${ }^{9,70}$ a specific themal load parameter value of 7.0 using LHG-5 glass doped with 3.5 wt-\% neodymium oxide and with a fluorescence lifetime of 240 microseconds. A pump pulse duration of 170 microseconds was used, which results in a fraction stored of 0.70 or 0.78 , for square or half-period sine pulse shape approximations, respectively. From Figure 4 , we predict a specific thermal load parameter value of 1.0 ; $x$ increases to 1.1 for $\eta_{\text {ext }}=0$ appropriate to the measurement condition. Both of these values are about a factor of two lower than that reported. The value cited by Eggleston and Byer may be fairiy inaccurate since it was deduced from a measurement of the themaliy induced depolarization. ${ }^{10}$ At the same time, significant uncertainties remain as to the actual ${ }^{4} F_{3 / 2}$ multiphonon decay rates in phosphate glasses. It is clear that multiphonan decay rates of candidate glasses should be accurately measured and that direct measurement of specific themal load parameter values should be carried out on the same glasses. In the meantime, the graphical data presented here may be used to guide our design analyses of high performance, high-arrerage-power Nd:glass lasers. 


\section{References}

1. J. L. Emmett, W. F. Krupke, and J. B. Trenho?me, "The Future Deveiopment of High-Power Sol id State Laser Systems," UCRL-53344, November 1982. See a1 so same authors, Sov. Jour. of Quantum Electronics, Vol. 10, January 1983.

2. R. A. Brandewie and C. L. Telk, "Quantum Efficiency of $\mathrm{Nd}^{3+}$ in Giass, Calcium Tungstate, and Ýttrium Aluminum Garnet," J.0.S.A., 57, $1221(1967)$.

3. C. Brecher, L. A. Riseberg, and M. J. Weber, App1. Phys. Letters, 30, 475 (1977); Phys. Rev. B, 18, 5799 (1978).

4. V. P. Gapantsev, M. R. Sirtlanov, A. K. Gromov, and A. A. Is ineev, Proc. International Conf. on Lasers ' 81 , New Orleans, LA, December 1981. STS Press, McLean, VA, pp. 763-768.

5. C. B. Layne, W. H. Lowdermitk, and M. J. Weber, Phys. Rev., 16, 10 (1977).

6. S. E. Stckowski, R. A. Saroyan, and M. J. Weber, "Nd-Doped Laser Glass Spectroscopic and Physical Properties," M-095, Lawrence Livermore Laboratory, 1978.

7. S. E. Stokowski, "Water and Concentration Quenching in LHG-B and LG-750," Internal Memorandum, Nova 55, Nova-82-1065, 55-B2-728, Juły $1,1982$.

8. S. E. Stokowski, "Ultraphosphate Glass Compositions, Status Report," Interna1 Memorandum, Nova-0pt-82-028, February 14, 1983.

9. J. M. Eggleston, T. J. Kane, J. Unternahrer, and R. L. Byer, Optics Letters, 7,405 (1982).

10. J. M. Eggleston, "Theoretical and Experimental Studies of Siab Geonetry Lasers," G. L. Report No. 3497, October 1982, Edward L. Ginzton Laboratory, Stanford University, CÁ. 
Table ?

ED-2 Heating Function

\begin{tabular}{|c|c|c|c|c|c|c|c|}
\hline \multirow[b]{2}{*}{$\underline{\rho}(w t-q)$} & \multirow[b]{2}{*}{$\tau_{f}(\mu \mathrm{sec})$} & \multirow[b]{2}{*}{$A_{f}\left(\sec ^{-1}\right)$} & \multirow[b]{2}{*}{$W_{43}+H_{q}(\rho)$} & \multicolumn{3}{|c|}{ Heat Distribution } & \multirow[b]{2}{*}{$H(G)$} \\
\hline & & & & $1060 \mathrm{~nm}$ & $1350 \mathrm{~nm}$ & $N R_{40}$ & \\
\hline $\begin{array}{l}0.03 \\
0.3 \\
1.0 \\
2.0 \\
3.0 \\
4.3 \\
6.0\end{array}$ & $\begin{array}{l}360 \\
360 \\
330 \\
309 \\
240 \\
199 \\
127\end{array}$ & $\begin{array}{l}2780 \\
2780 \\
3030 \\
3236 \\
4157 \\
5026 \\
7874\end{array}$ & $\begin{array}{r}200 \\
200 \\
452 \\
658 \\
1590 \\
2447 \\
5296\end{array}$ & $\begin{array}{l}0.110 \\
0.110 \\
0.091 \\
0.085 \\
0.066 \\
0.055 \\
0.035\end{array}$ & $\begin{array}{l}0.042 \\
0.042 \\
0.034 \\
0.032 \\
0.025 \\
0.027 \\
0.013\end{array}$ & $\begin{array}{l}0.088 \\
0.088 \\
0.18 ? \\
0.248 \\
0.465 \\
0.594 \\
0.821\end{array}$ & $\begin{array}{l}0.230 \\
0.230 \\
0.307 \\
0.365 \\
0.556 \\
0.670 \\
0.869\end{array}$ \\
\hline
\end{tabular}

$$
A_{\text {rad }}=2578 \mathrm{sec}^{-1} \quad T_{\text {rad }}=388 \mu \mathrm{sec} \quad W_{43}=200 \mathrm{sec}^{-1}
$$

Table 2

LG-650 Heating Function

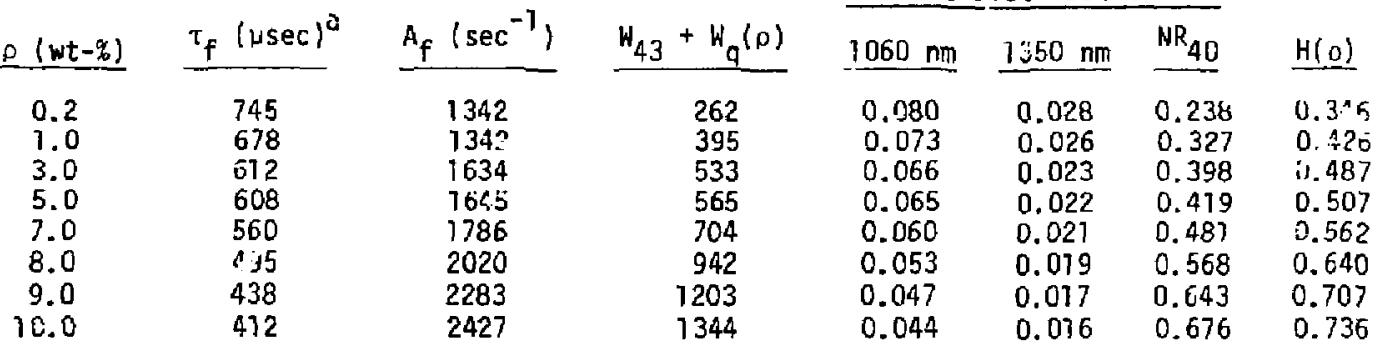

$$
A_{\text {rad }}=1080 \mathrm{sec}^{-1} \quad \tau_{\text {rad }}=926 \mathrm{usec} \quad W_{43}=262 \mathrm{sec}^{-1} \quad a_{\tau_{f}}=\left\{\tau_{1}+\tau_{2}\right\} / 2
$$


Table 3

LHG-5 Heating Function

\begin{tabular}{|c|c|c|c|c|c|c|c|}
\hline \multirow[b]{2}{*}{$\underline{\rho}(w t-g)$} & \multirow[b]{2}{*}{$\tau_{f}(\text { (ustc) })^{\pi}$} & \multirow[b]{2}{*}{$A_{f}\left(\sec ^{-1}\right)$} & \multirow[b]{2}{*}{$\ddot{H}_{\Delta 3}+H_{q}(0)$} & \multicolumn{3}{|c|}{ Heat Distribution } & \multirow[b]{2}{*}{$H(\underline{p})$} \\
\hline & & & & $1060 \mathrm{~nm}$ & $1350 \mathrm{~nm}$ & $\mathrm{NR}_{40}$ & \\
\hline $\begin{array}{l}0.3 \\
1.0 \\
3.3 \\
3.5 \\
6.0\end{array}$ & $\begin{array}{l}319 \\
318 \\
275 \\
260 \\
199\end{array}$ & $\begin{array}{l}3135 \\
3134 \\
3536 \\
3846 \\
50025\end{array}$ & $\begin{array}{r}261 \\
271 \\
763 \\
972 \\
2152\end{array}$ & $\begin{array}{l}0.101 \\
0.101 \\
0.087 \\
0.082 \\
0.063\end{array}$ & $\begin{array}{l}0.036 \\
0.036 \\
0.031 \\
0.030 \\
0.023\end{array}$ & $\begin{array}{l}0.102 \\
0.105 \\
0.256 \\
0.302 \\
0.522\end{array}$ & $\begin{array}{l}0.239 \\
0.242 \\
0.374 \\
0.415 \\
0.608\end{array}$ \\
\hline$A_{r}$ & $2874 \mathrm{sec}^{-}$ & $T_{\text {rad }}=348$ & $W_{43}$ & $\sec ^{-1}$ & $\mathrm{a}_{\mathrm{T}_{f}}=$ & $\left.+T_{2}\right) / 2$ & \\
\hline
\end{tabular}

Table 4

LG-750 Heating Function

\begin{tabular}{|c|c|c|c|c|c|c|c|}
\hline \multirow[b]{2}{*}{$=(w t-\%)$} & \multirow[b]{2}{*}{$\tau_{f}(\mu \mathrm{sec})$} & \multirow[b]{2}{*}{$A_{f}\left(\sec ^{-1}\right)$} & \multirow[b]{2}{*}{$W_{43}+W_{q}(\rho)$} & \multicolumn{3}{|c|}{ Heat Distribution } & \multirow[b]{2}{*}{$A(0)$} \\
\hline & & & & $1060 \mathrm{~nm}$ & $1350 \mathrm{~nm}$ & $N_{40}^{N}$ & \\
\hline $\begin{array}{r}0.5 \\
2.0 \\
3.0 \\
4.0 \\
6.0 \\
8.0 \\
10.0\end{array}$ & $\begin{array}{l}356 \\
336 \\
315 \\
289 \\
234 \\
185 \\
145\end{array}$ & $\begin{array}{l}2812 \\
2972 \\
3776 \\
3461 \\
4277 \\
5419 \\
6887\end{array}$ & $\begin{array}{r}745 \\
305 \\
509 \\
795 \\
1670 \\
2752 \\
4220\end{array}$ & $\begin{array}{l}0.104 \\
0.099 \\
0.092 \\
0.085 \\
0.069 \\
0.054 \\
0.043\end{array}$ & $\begin{array}{l}0.040 \\
0.037 \\
0.035 \\
0.032 \\
0.026 \\
0.021 \\
0.016\end{array}$ & $\begin{array}{l}0.063 \\
0.125 \\
0.196 \\
0.280 \\
0.459 \\
0.620 \\
0.748\end{array}$ & $\begin{array}{l}0.207 \\
0.261 \\
0.323 \\
0.392 \\
0.554 \\
0.695 \\
0.807\end{array}$ \\
\hline & Ara & $567 \mathrm{sec}^{-1}$ & ${ }^{\mathrm{T}}$ rad $=356$ & $W_{4}$ & 145 & & \\
\hline
\end{tabular}


Table 5

S-16 Heating Function ${ }^{a}$

Heat Distribution

\begin{tabular}{|c|c|c|c|c|c|c|c|}
\hline \multirow[b]{2}{*}{$\rho(w t-z)$} & \multirow[b]{2}{*}{$\tau_{f}(\mu s \in c)$} & \multirow[b]{2}{*}{$A_{f}\left(\sec ^{-1}\right)$} & \multirow[b]{2}{*}{$w_{43}+w_{q}(p)$} & & \multirow[b]{2}{*}{$\mathrm{H}(\rho)$} \\
\hline & & & & $1060 \mathrm{~nm}$ & $1350 \mathrm{~nm}$ & $\mathrm{NR}_{40}$ & \\
\hline $\begin{array}{r}0.0 \\
0.5 \\
5.0 \\
10.4 \\
22.7\end{array}$ & $\begin{array}{c}(365) \\
357 \\
272 \\
194 \\
99\end{array}$ & $\begin{array}{r}2740 \\
2801 \\
3677 \\
5155 \\
10,100\end{array}$ & $\begin{array}{r}0 \\
61 \\
937 \\
2475 \\
7361\end{array}$ & $\begin{array}{l}0.110 \\
0.108 \\
0.082 \\
0.058 \\
0.030\end{array}$ & $\begin{array}{l}0.042 \\
0.041 \\
0.031 \\
0.022 \\
0.011\end{array}$ & $\begin{array}{l}0.000 \\
0.027 \\
0.311 \\
0.572 \\
0.889\end{array}$ & $\begin{array}{l}0.152 \\
0.178 \\
0.424 \\
0.652 \\
0.930\end{array}$ \\
\hline
\end{tabular}

$$
A_{\text {rad }}=2740 \mathrm{sec}^{-1} \quad \tau_{\text {rad }}=365 \mathrm{\mu sec} \quad W_{43}=0 \quad \text { aReference } 8
$$


KINETICS OF Nd:GLASS $(\rho=0)$

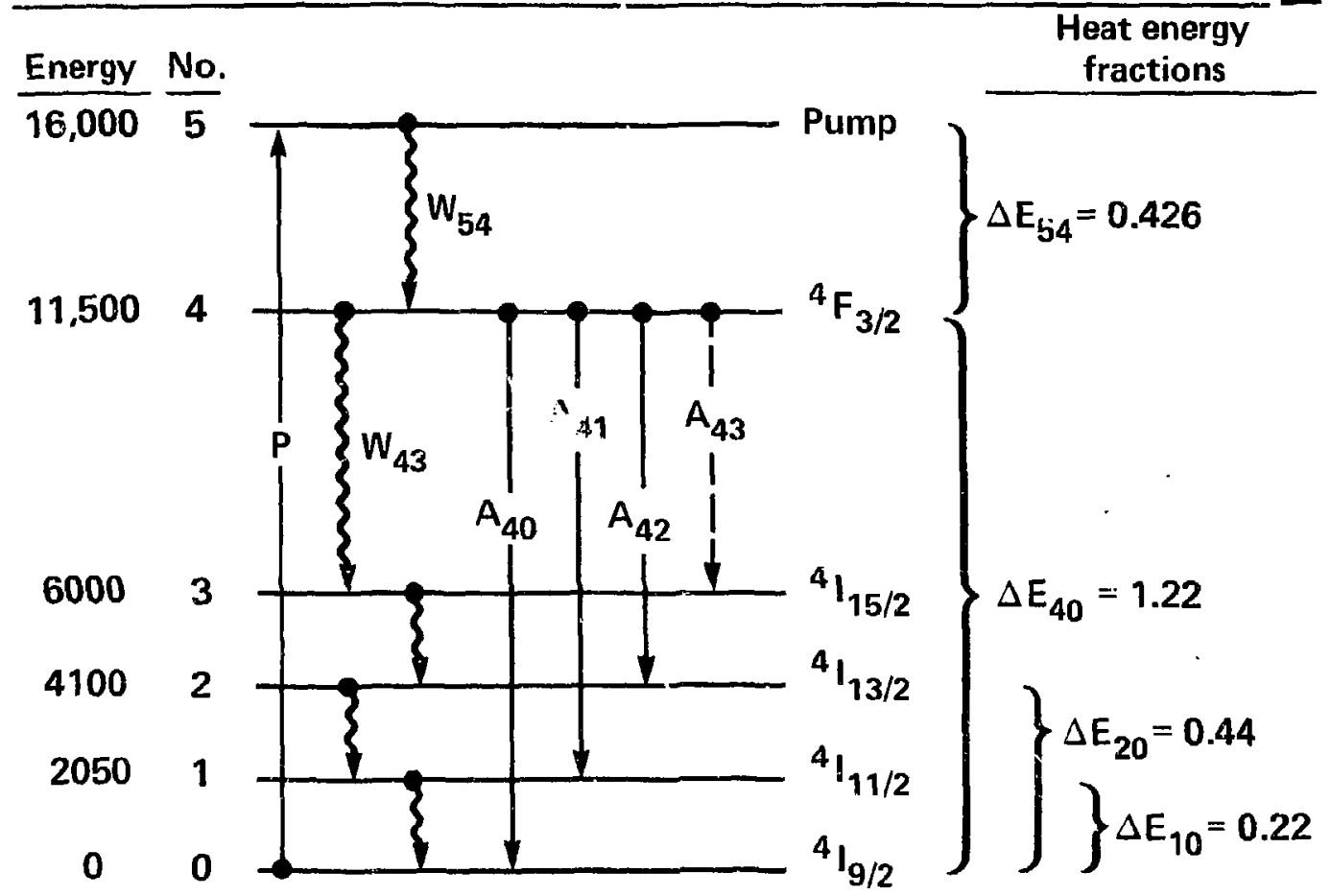

02-50-0483-1078

Figure 1: Multiohonon Decay Heating Channe1s in idd:Glass (heat energy fractions in units of $9400 \mathrm{~cm}^{-1}$ ) 


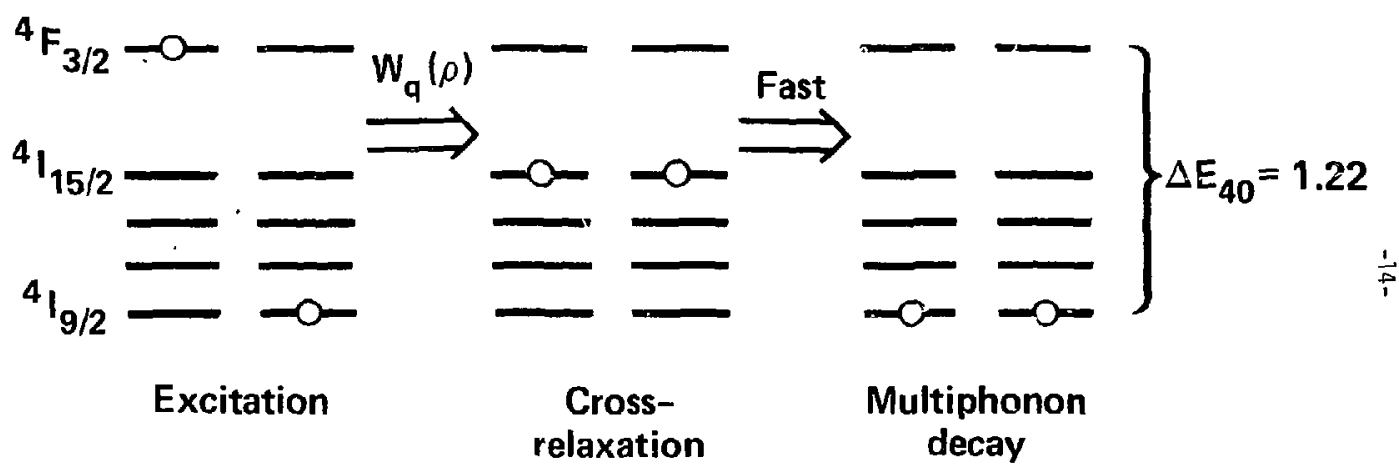

Fiqure 2: Ion-Ion Cross-Relaxation Heating Channel in Nd:Slass (heat energy fraction $\Delta E_{4 C}$ in units of $9400 \mathrm{~cm}^{-1}$ ) 


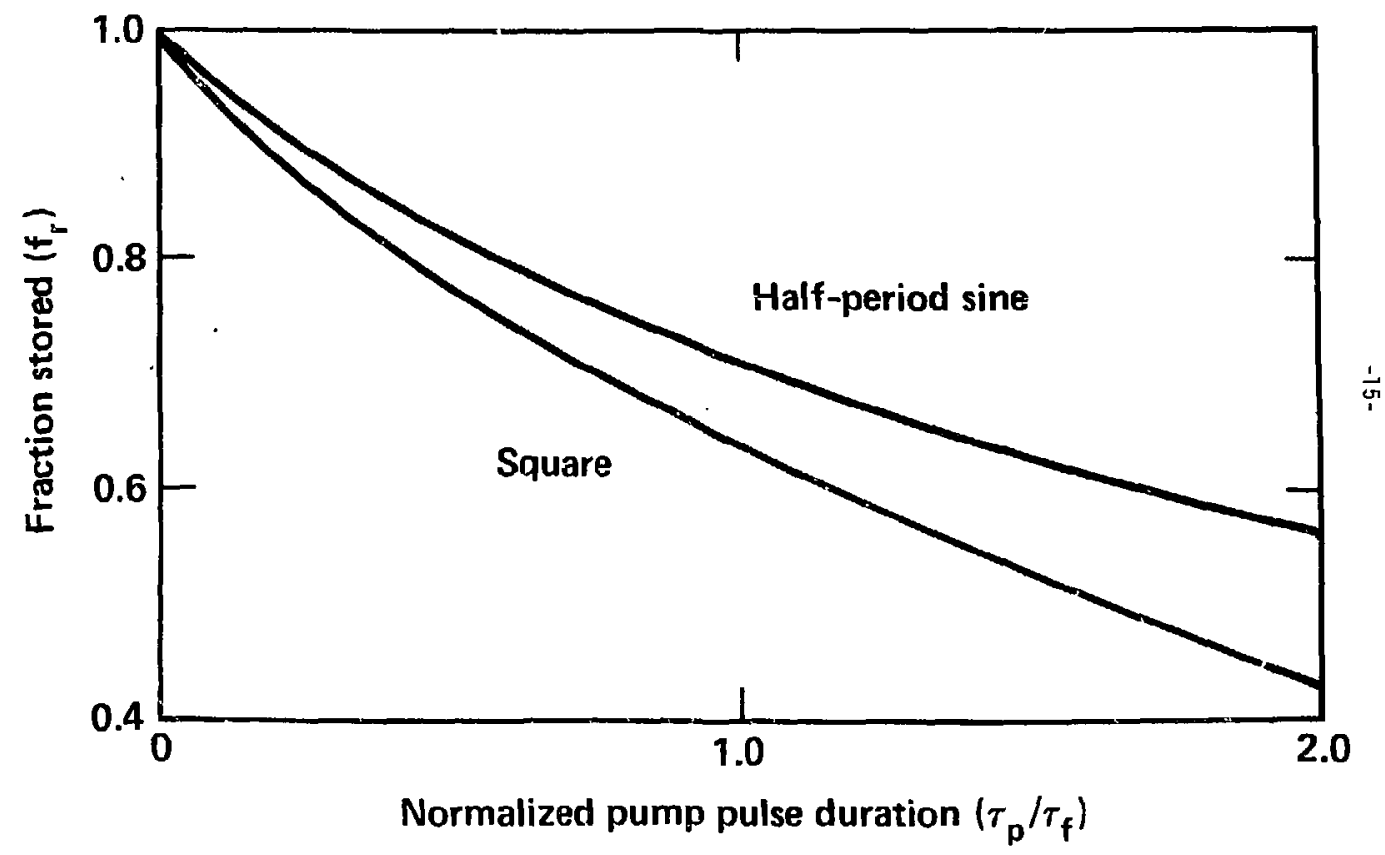



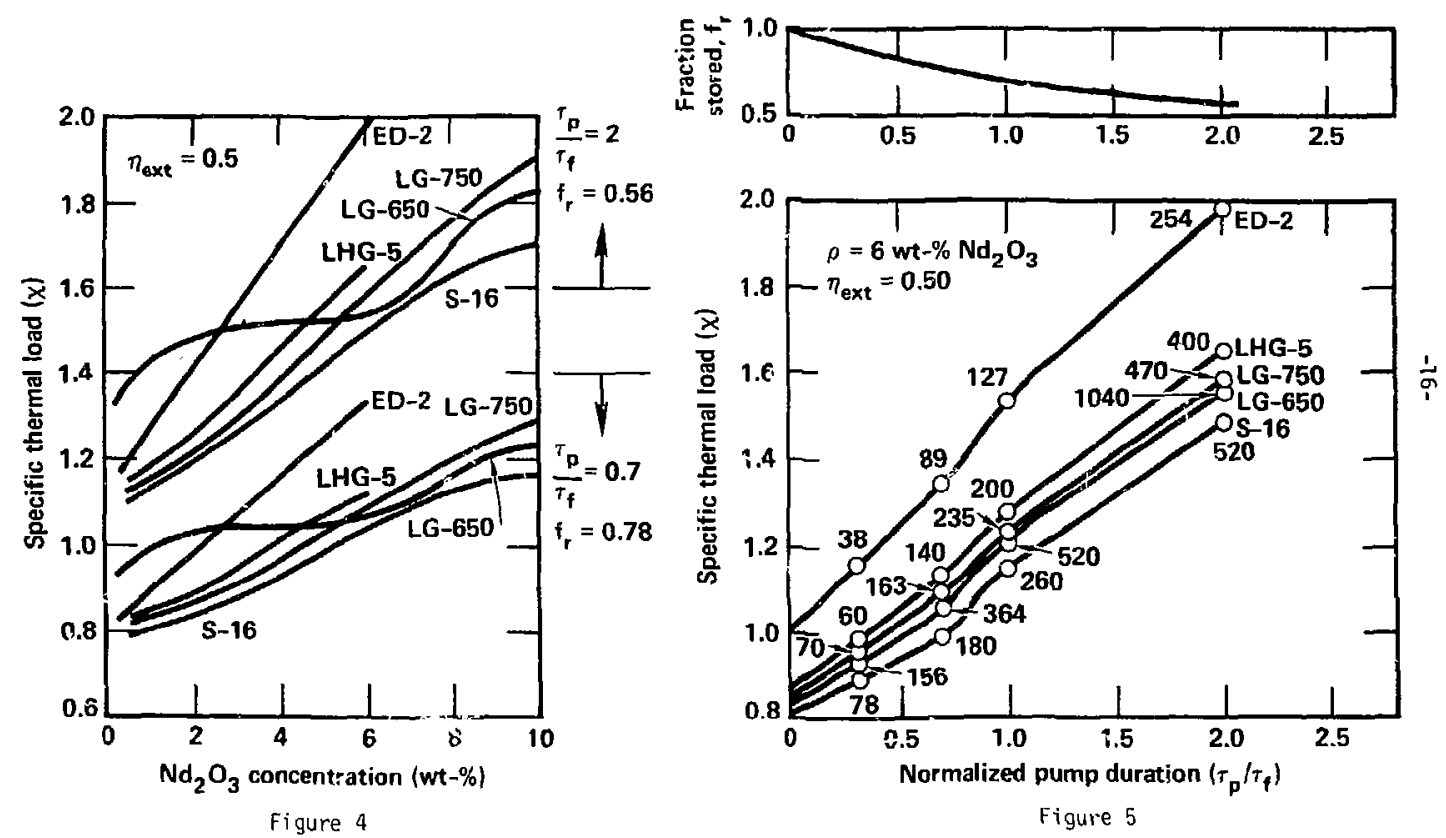

Figure 5

02.50 .0483 .1081 
SPECIFIC THERMAL LOAD $\chi$ vs $\eta_{\text {ext }}$

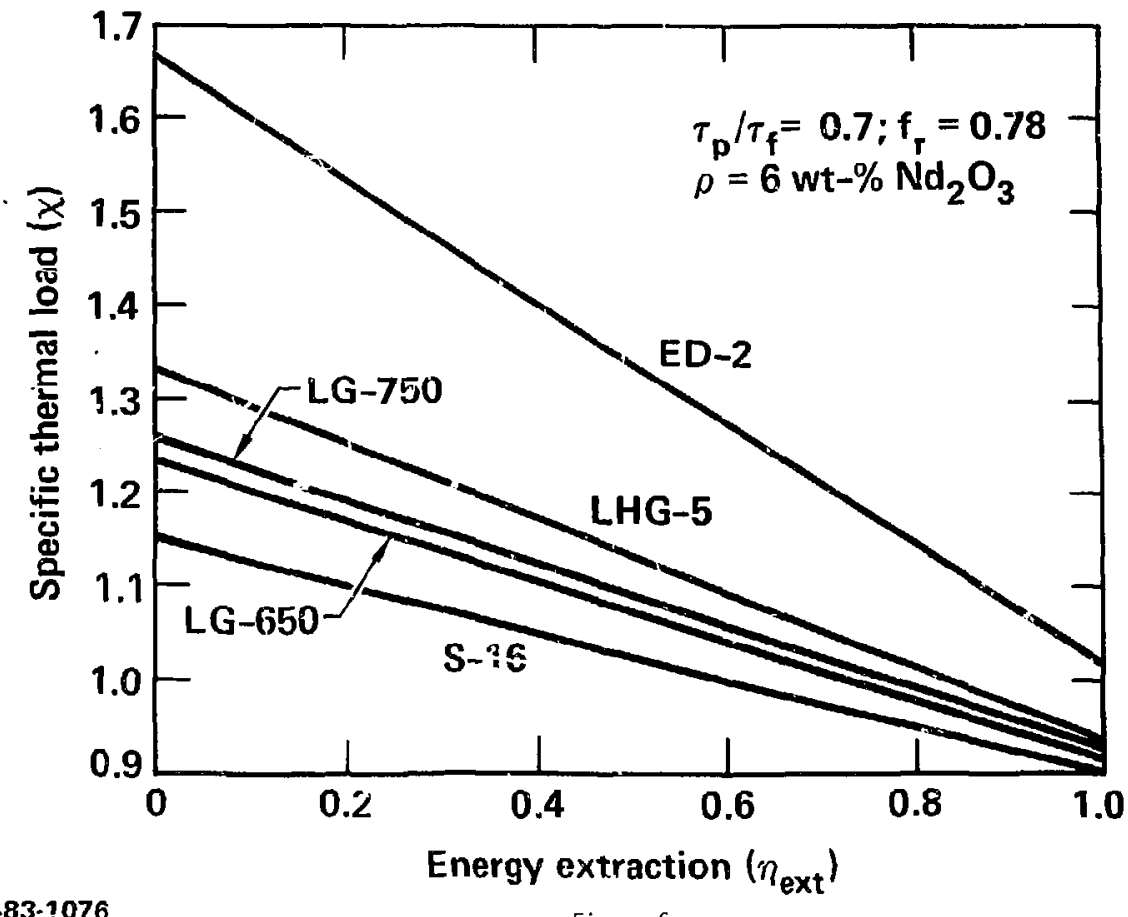


$-\mathrm{dx} / \mathrm{d} \eta_{\text {ext }}$ vs $\rho$

14

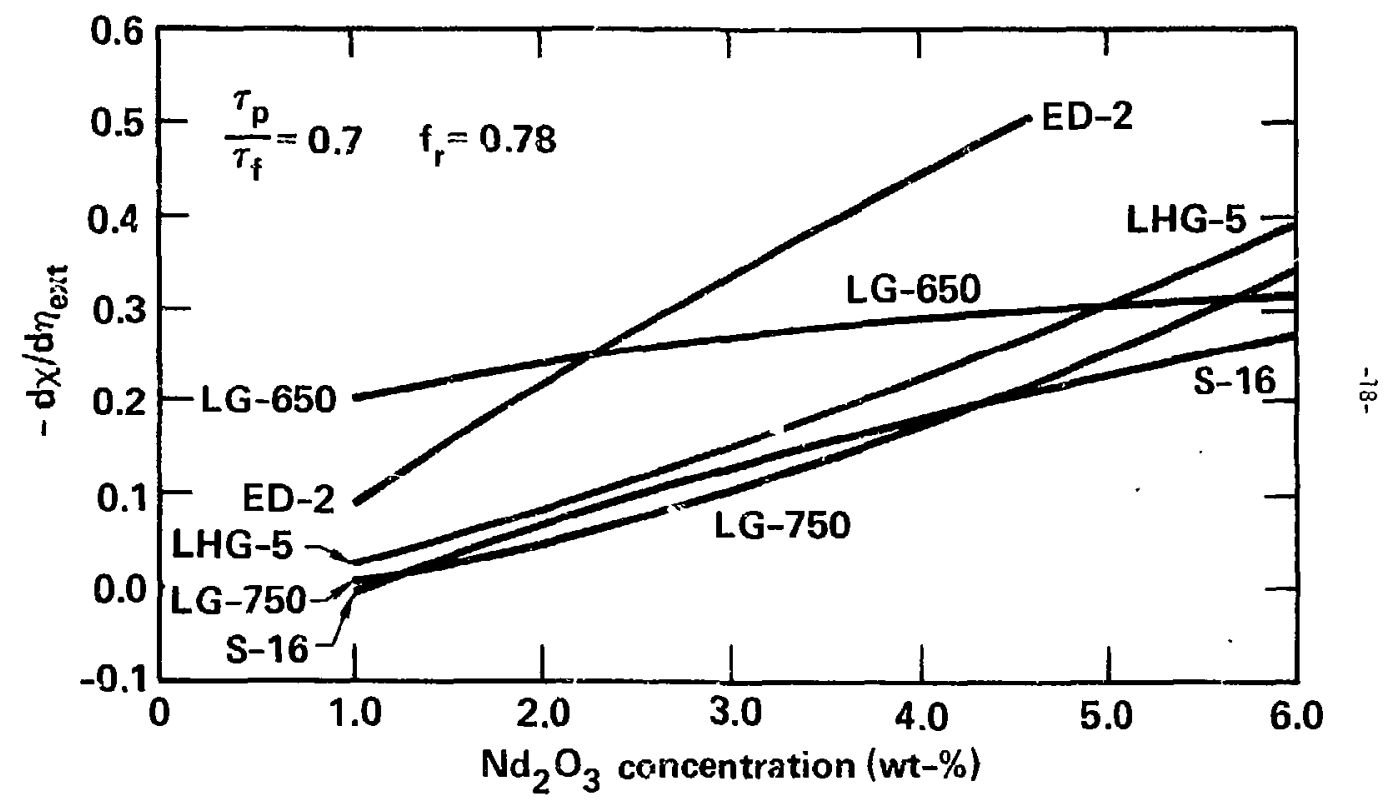




\section{DISCLALMER}

This report was prepared as an account of work sponsored by an agency of the United States Government. Neither the United States Government nor any agency thereof, nor any of their employes, makes any waranty, express or implied, or assumes any legal liability or responsibjlity for the accuracy, completeness, or usefulness of any information, apparatus, producl, or process disclosp.d, or represents that its use would not infringe privately owned rights. Reference herein to any specific commercial prorluct, process, or service by trade name, trademark, manufacturer, or atherwise does not necessanily constitute or imply its endorsement, recommendation, or favoring by the United States Government or any agency thereof. The views and opinions of authors expressed herein do not necessarily state or reflec! those of the United States Government or any agency thereof. 